\title{
Intrinsically Elastic Robots: the Key to Human Like Performance
}

\author{
Sami Haddadin, Felix Huber, Kai Krieger, Roman Weitschat, Alin Albu-Schäffer, Sebastian Wolf, Werner Friedl, \\ Markus Grebenstein, Florian Petit, Jens Reinecke, Roberto Lampariello
}

\begin{abstract}
Intrinsically elastic robots, which technically implement some key characteristics of the human muskoskeletal system, have become a major research topic in nowadays robotics. These novel devices open up entirely new control approaches. They base on temporary storage of potential energy and its timed transformation into kinetic energy. In legged locomotion, such considerations have been a common tool for unveiling the respective fundamental physical processes. However, in arm control, elasticities were typically considered parasitic. In this video we outline our efforts in exploiting the inherent capabilities of intrinsically elastic robots in order to bring them closer to human performance. Instead of applying purely kinematic learing-by-demonstration approaches, which are certainly suboptimal, we argue for using model based techniques in order to optimally exploit the system dynamics such that highly dynamic motion and manipulation capabilities can be achieved. In particular, the explicit use of elasticities as temporary energy tanks can be fully exploited, if they are modeled adequately as an integral part of the mechanism. We also believe that such approaches can substantially contribute to the understanding of human motion biomechanics.
\end{abstract}

\section{INTRODUCTION}

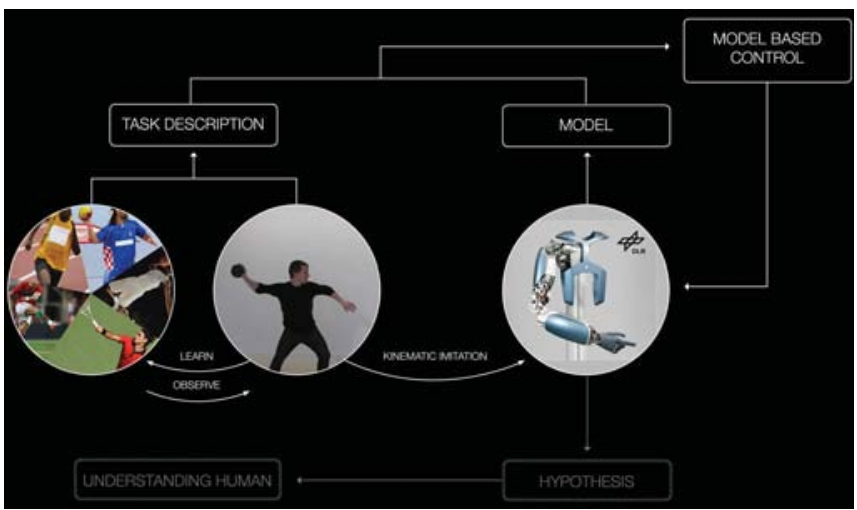

Fig. 1. How can robots achieve human like performance?

The elastic muskoskeletal system of the human enables remarkable robustness and performance [1]. Based on continuous observation and learning, humans are able to optimize their motion schemes such that the exploitation of their inherent flexible dynamics is maximized, see Fig. 1. Even though robotic systems outperform humans in terms of most automation performance measures such as repeatability, endurance, and accuracy, they are hopelessly inferior to humans in terms of maximum speed and dynamic motion capability. Consequently, large efforts are put into designing and controlling systems that might come closer to human performance. In particular, intrinsically elastic arms are sought to overcome the limitations of classical rigid robots. At DLR, e.g., a new variable stiffness hand-arm system (HASy) was

all authors are is with the Robotics and Mechatronics Center, DLR - German Aerospace Center, Wessling, Germany sami.haddadin@dlr. de, This work has been partially funded by the European Commission's Sixth Framework Programme as part of the project SAPHARI under grant no. 287513. developed [2]. It is equipped with two actuators and an adjustable elastic transmission element per joint, allowing simultaneous control of position and stiffness. This system is able to safely interact with humans if being controlled adequately [3]. Furthermore, the joint compliance protects the robot from getting damaged due to collisions [4], [5]. However, the certainly most interesting property of intrinsically compliant robots is their capability to execute highly dynamic motions by explicitely using the elastic joints as temporary energy tanks.

Currently, teaching human like motions to robots is typically carried out via kinematic imitation approaches [6], [7], [8]. These well established schemes are, however, suboptimal and will never exploit the inherent capabilities of the aforementioned systems. Presumably, it will not even come close to what might be achievable in principle. In contrast, the combination of a formal task description, an accurate robot dynamics model, and a suitable model based control approach are the rigorous way of optimally solving the given task [9], [10] under the premise of the robot's characteristics. In this line of thinking, we laid the foundations on optimally achieving explosive motions by temporary storage of potential energy and its timed transformation into kinetic energy [4], [11], [12].

Also during human cyclic manipulation such as dribbling the use of the elastic finger and wrist properties is apparent [13], see Fig. 2. This problem can be formulated in terms of hybrid systems theory [14]. The developed formulation served us to investigate the effects of elastic energy storage and release for ball dribbling in terms of cycle stability. We based the analysis on error evolution, peak power performance during hand contact, and task robustness with respect to varying hand stiffness. As the ball can only be controlled during contact, an intrinsically elastic hand extends the contact time and improves the energetic characteristics of the process. Generally, one can derive the optimal finger compliance in terms of cycle robustness and power consumption for blind dribbling with force feedback in the wrist only.

Based on this principal analysis of elastic end-effectors and manipulators, we designed an optimal motion framework for fully elastic robots [12], see Fig. 3. Generating optimal motions under the premise of the complex elastic dynamics, an optimal control problem has to be solved offline for a given task, which we call prototypical optimal control problem (POCP). Since the full elastic dynamics are modeled, the according motion optimally exploits them in the sense of the chosen cost function. In order to generate optimal motions in real-time, we solve the respective POCP e.g. for a set of goal states offline. These optimal trajectories are learned by a dynamical system (Dynamic Movement Primitive, DMP) and can then be generalized instantaneously to a new goal state with a cost metric based distance measure. This framework can, for example, be used to execute near-optimal explosive 

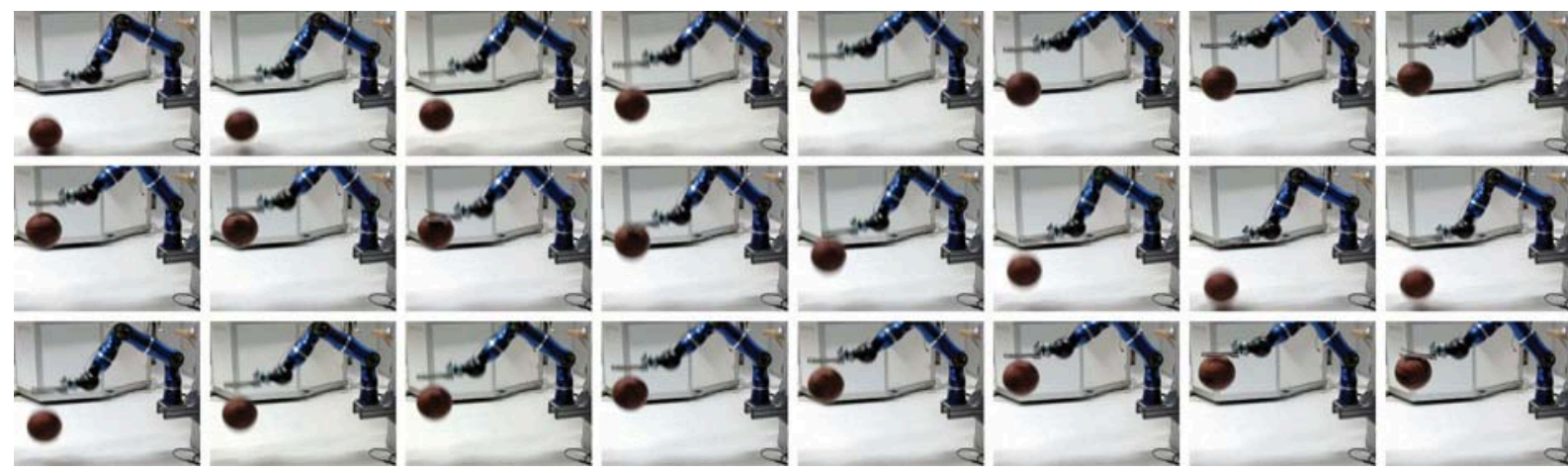

Fig. 2. Snapshots from basketball dribbling with the LWR-III that is equipped with an elastic hand.
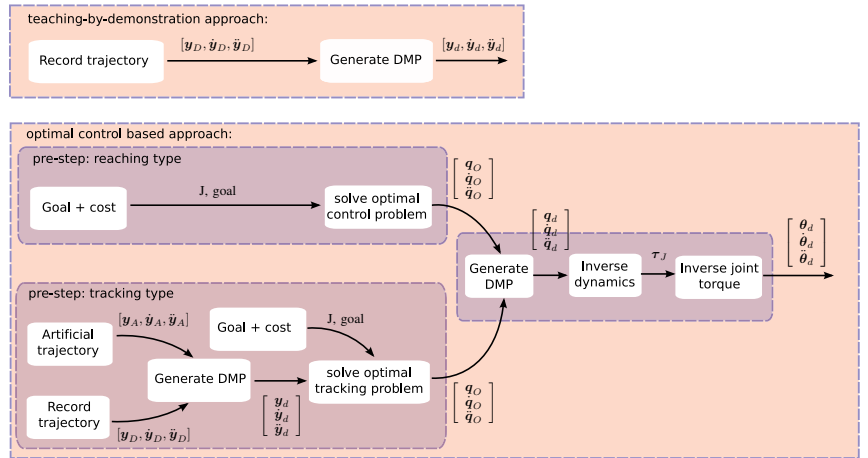

Fig. 3. Methodological overview of the optimal motion framework. In contrast to the classical learning-by-demonstration paradigm (upper), we start from solving complex optimal control problems and use these dynamically optimal trajectories as learning input. We encode them into an optmimized dynamical system in terms of Dynamic Movement Primitives (DMPs) and use a metric based on the cost function or geometric distance for selecting a near-optimal parameter set in real-time for the generalizing step Worth noticing is that our framework includes learning-by-demonstration approaches as well.

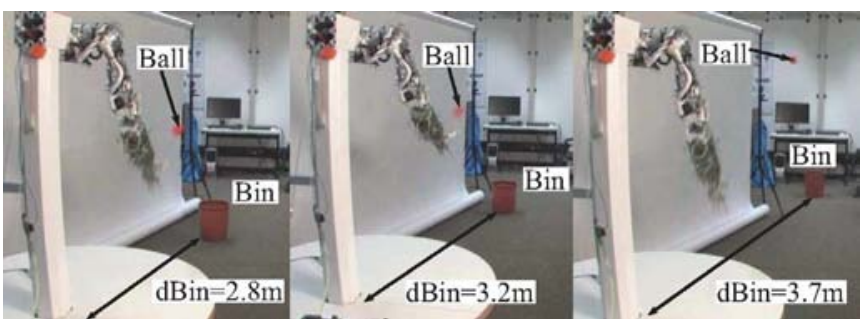

Fig. 4. Throwing sequence for varying target (bin). The bin is detected with a Kinect sensor and the distance $d_{B i n}$ parameterizes the goal $\mathbf{g}$ (Bin). All trials were successful scores.

motions such as throwing a ball in a feedback loop, see Fig. 4. In the video, the task is to throw a ball into a bin. The bin is tracked by a camera system and the dynamically optimal motions are generated online $(3 \mathrm{kHz})$.

\section{CONCLUSiON}

In this video, we presented our work on controlling intrinsically compliant robots such that human like performance in terms of dynamics can be approached. The explicit model based use of elasticity for temporary energy storage is the key to these achievements. The developed methods enable robots to optimally exploit their inherent capability and in direct consequence achieve tasks that were not possible before. With the developed frameworks, tasks such as blind basketball dribbling with optimally robust and power minimizing hand elasticities, or near-optimal real-time execution of explosive motions for throwing a ball become possible. Clearly, the understanding of how elasticities can be considered a fundamental feature instead of being a drawback for arm control needs a paradigm shift. Our results show that one has to enlarge the set of well established relevant control problems for these elastic devices in order to be able to target for the human antetype's performance. Furthermore, we also believe that the thorough understanding of the dynamics and control of intrinsically elastic robots will give us better insight into the underlying mechanisms of human motion. This is due to the conceptual similarity between elastic robots and the human muskoskeletal system.

\section{REFERENCES}

[1] I. Herman, Physics of the Human Body. Springer Verlag, 2007

[2] M. Grebenstein et al., "The DLR hand arm system," 2011, pp. 31753182.

[3] S. Haddadin, A. Albu-Schäffer, O. Eiberger, and G. Hirzinger, "New insights concerning intrinsic joint elasticity for safety," in IEEE/RSJ Int. Conf. on Intelligent Robots and Systems (IROS2010), Taipeh, Taiwan, 2010.

[4] S. Haddadin, T. Laue, U. Frese, S. Wolf, A. Albu-Schäffer, and G. Hirzinger, "Kick it with elasticity: Requirements for 2050," Robotics and Autonomous Systems, vol. 57, pp. 761-775, 2009.

[5] G. H. R. S. Markus Grebenstein, Maxime Chalon, "Antagonistically, driven finger design for the anthropomorphic dlr hand arm system," HUMANOIDS, 2010.

[6] K. Muelling, J. Kober, and J. Peters, "A biomimetic approach to robot table tennis," Adaptive Behavior Journal, vol. 19, no. 5, pp. 359-376, 2011.

[7] P. Pastor, H. Hoffmann, T. Asfour, and S. Schaal, "Learning and generalization of motor skills by learning from demonstration," in IEEE Int. Conf. on Robotics and Automation, 2009, pp. 763-768.

[8] J. A. Ijspeert, J. Nakanishi, and S. Schaal, "Learning rhythmic movements by demonstration using nonlinear oscillators," in IEEE/RSJ Int. Conf. on Intelligent Robots and Systems, 2002, pp. 958-963.

[9] W. Coppel, Mathematical Control Theory, ser. Lecture Notes in Mathematics, 1978, no. 680.

[10] S. Ge and Z. Sun, Switched linear systems: Control and design, ser. Communications and control engineering series. Springer, 2005.

[11] S. Haddadin, M. Weis, S. Wolf, and A. Albu-Schäffer, "Optimal control for maximizing link velocity of robotic variable stiffness joints," 2011 IFAC World Congress (IFAC2011), Milano, Italy, pp. 6863-6871.

[12] S. Haddadin, F. Huber, and A. Albu-Schäffer, "Optimal control for exploiting the natural dynamics of variable stiffness robots," in IEEE Int. Conf. on Robotics and Automation, 2012.

[13] S. Haddadin, K. Krieger, and A. Albu-Schäffer, "Exploiting elastic energy storage for cyclic manipulation: An analysis for basketball dribbling with an anthropomorphic robot," in accepted to: IEE/RSJ International Conference on Intelligent Robots and Systems, San Francisco, USA, 2011.

[14] — - "Exploiting elastic energy storage for cyclic manipulation: Modeling, stability, and observations for dribbling," in IEEE Conference on Decision and Control, Orlando, USA, 2011. 